\title{
HIV swiftly guts the immune system
}

\author{
Ronald S Veazey \& Andrew A Lackner
}

Two studies show that SIV directly kills massive numbers of immune cells in the gut within days of infection. The results come on the heels of similarly dramatic findings for HIV, and could radically shift the focus of HIV research and therapy.

For years, some AIDS researchers have suspected that the search for answers has been focusing in the wrong place-that the gut and other mucosal tissues, and not the blood, is the major site of HIV infection and CD4+ T cell loss. Two recent reports in Nature should put doubts about this view to rest, and further emphasize the speed with which HIV disarms the immune system. The findings have profound implications for HIV research and therapy.

Mattapallil et al. and Li et al. both show that simian immunodeficiency virus (SIV) rapidly and selectively infects and destroys memory $\mathrm{CD}^{+} \mathrm{T}$ cells within days of infection $^{1,2}$. This results in loss of most of the 'functioning' (i.e., 'memory') $\mathrm{CD} 4^{+} \mathrm{CCR} 5^{+}$ $\mathrm{T}$ cells in the body. The major focus of this destruction is in mucosal tissues, where most of T cells expressing CD4 and CCR5-the receptors for HIV attachment and entrywith a 'memory' phenotype reside.

Although previous reports have shown massive loss of memory CCR5+ CD4+ $\mathrm{T}$ cells in SIV-infected macaques ${ }^{3,4}$, the major importance of the new studies is that they both describe selective infection of these cells by SIV. The findings strongly support the notion that these cells are killed by direct, virally mediated destruction rather than through bystander effects or loss of CD4+ T cell production.

Such findings have eluded researchers for years, mainly because most studies examined only peripheral blood or lymph nodes, sites that have very few of these viral 'target' cells.

The authors are at the Tulane National Primate Research Center, Tulane University School of Medicine, Covington, Louisiana 70433, USA. e-mail: rveazey@tulane.edu

a Primary lymphoid tissues b Inductive lymphoid tissues

C Effector lymphoid tissues

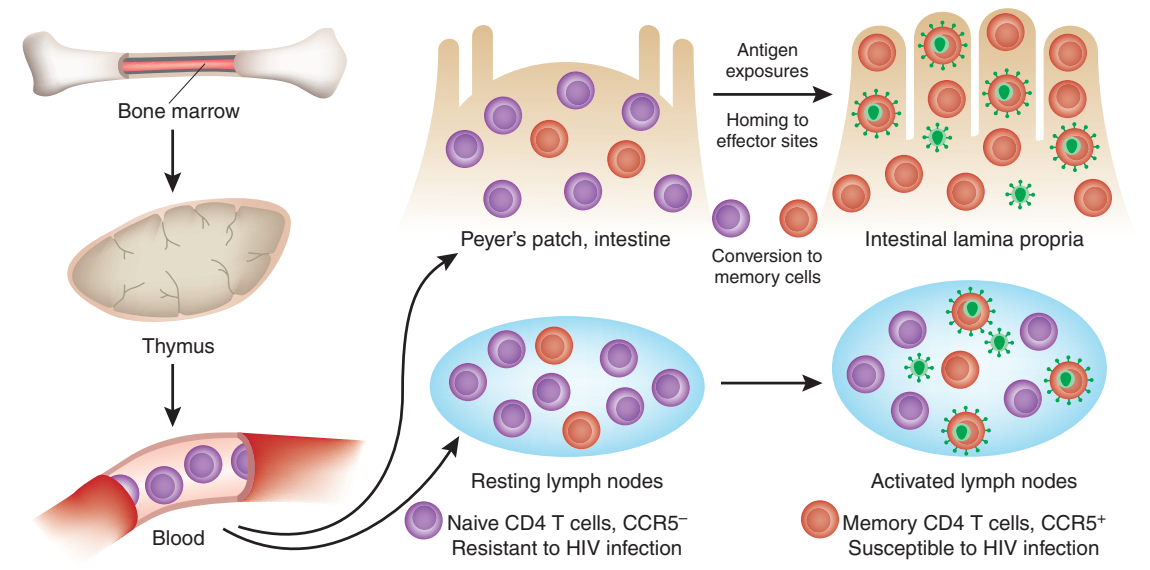

Figure 1 HIV's lair. Early HIV infection and replication mainly occurs in mucosal effector lymphoid tissues, as these contain the vast majority of the body's memory CD4 ${ }^{+} \mathrm{CCR} 5^{+} \mathrm{T}$ cells. (a,b) Naive T cells emerge from the thymus and home to inductive lymphoid tissues as 'resting, naive' cells until they encounter their cognate antigen. (c) Once activatd by antigen, these cells rapidly migrate and home to effector lymphoid tissues and become 'memory' cells that coexpress high levels of CCR5. Thus, most of the CD4 cells that are permissive to HIV infection (memory $\mathrm{CD} 4^{+} \mathrm{CCR} 5^{+}$cells) are located in effector lymphoid tissues. Activated lymph nodes may contain increased numbers of activated CCR $5^{+}$memory CD4 cells in chronically infected individuals, but it is increasingly clear that most HIV replication occurs in mucosal tissues, particularly in acute infection.

The inability to detect sufficient numbers of infected $\mathrm{CD} 4^{+} \mathrm{T}$ cells in the peripheral blood led many to speculate that 'bystander' apoptosis of uninfected cells must underlie the loss of $\mathrm{CD}^{+} \mathrm{T}$ cells in AIDS.

Mattapallil et al. and Li et al. provide evidence that direct infection of memory $\mathrm{CD}^{+} \mathrm{T}$ cells is instead the major mechanism responsible for the loss of these cells in HIV-infected individuals, at least in primary infection. Moreover, recent studies showed that this loss of intestinal memory $\mathrm{CD}^{+}{ }^{+} \mathrm{CCR}^{+} \mathrm{T}$ cells occurs just as profoundly, rapidly and persistently in HIV-infected individuals as it does in SIV-infected macaques ${ }^{5,6}$, further verifying the nonhuman primate model, and providing additional significance to the work in Nature.

Combined, all these recent findings support the hypothesis, originally proposed in 1998, that the mucosal immune system in general, and the intestinal immune system in particular, is the major site of viral 

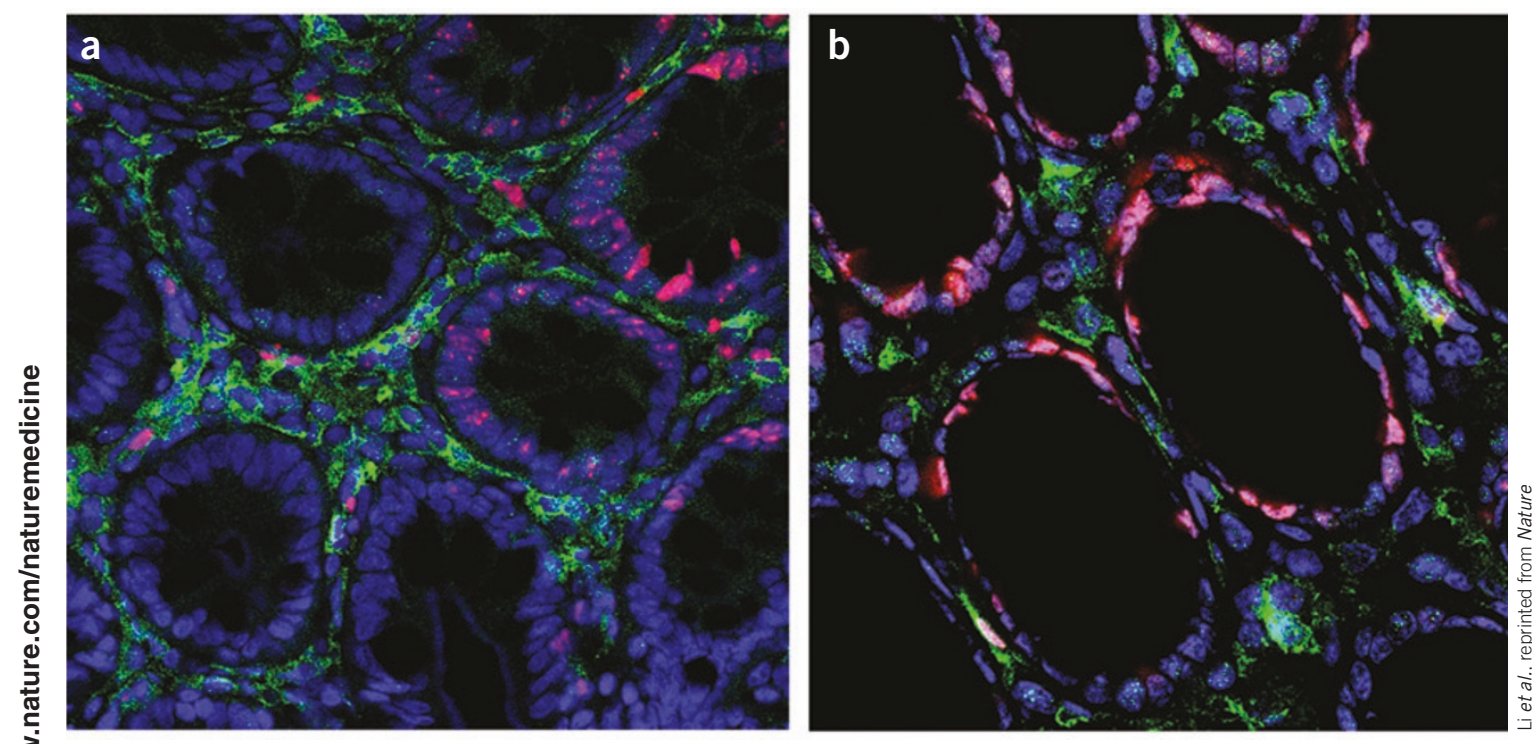

Figure 2 Depleting the gut. (a) Four days after SIV infection, the lamina propria of the intestine still contains a robust population of memory $\mathrm{CD} 4^{+} \mathrm{T}$ cells (green). (b) By ten days after infection many of these $\mathrm{CD}^{+}$cells have been destroyed. Ki-67+ (proliferating cells) are in red, and cell nuclei are in blue.

replication, persistence and $\mathrm{CD} 4^{+} \mathrm{T}$ cell loss in HIV-infected individuals ${ }^{7}$.

Additional evidence for this hypothesis comes from both HIV and SIV studies. In 2003, Peter Anton and colleagues showed that viral replication is active and ongoing in the intestine of HIV-infected individuals on therapy ${ }^{8}$. Louis Picker and colleagues also recently showed that the rate of turnover of memory $\mathrm{CD}^{+} \mathrm{T}$ cells in mucosal tissues determines the rate of progression to disease in SIV infection. Macaques that did not replenish these cells in mucosal tissues experienced rapid onset of opportunistic infections and the development of AIDS, whereas those with increased turnover of these cells had delayed progression to disease ${ }^{9}$.

Combined, these results present a unifying hypothesis for the pathogenesis of HIV infection that centers on these mucosal viral 'target' cells. Persistent HIV infection and replication in mucosal tissues, driven by continual turnover of memory CD4 ${ }^{+}$ $\mathrm{T}$ cells in effector lymphoid tissues such as the intestinal lamina propria (Fig. 1), is likely to be the major source of viral replication, persistence and continual $\mathrm{CD} 4^{+} \mathrm{T}$ cell loss in HIV-infected individuals, even in those on antiretroviral therapy with undetectable virus in blood.

The implications of these recent findings extend to pathogenesis, treatment and vaccine strategies. It should now be clear that trying to understand the pathogenesis of AIDS by examining events in peripheral blood is difficult at best and misleading at worst because the primary targets of infection are in mucosal tissues, not blood. It is also apparent that HIV infection essentially eliminates memory responses of
$\mathrm{CD}^{+}{ }^{+} \mathrm{T}$ cells to HIV and other potential opportunistic infections within days, rather than years, of infection. Although opportunistic infections are generally thought of as late events, in careful studies in nonhuman primates it is clear that subclinical opportunistic infections occur very early in infection ${ }^{10}$. Furthermore, it is important to remember that the destruction of memory $\mathrm{CD}^{+} \mathrm{T}$ cells is a dynamic event, with the body making a valiant but futile effort to replace these cells. Thus, although the rapid loss of these cells is dramatic, it is not immediately catastrophic.

These studies also indicate that treatment strategies aimed at stopping or delaying treatment in HIV-infected individuals based primarily on peripheral blood viral loads, $\mathrm{CD} 4^{+} \mathrm{T}$ cell counts, or both, are misguided. The loss of intestinal $\mathrm{CD} 4^{+} \mathrm{T}$ cells is rapid, profound and continual-regardless of what is occurring in the peripheral blood $^{1-7}$. In light of this, we believe it is important to treat HIV-infected individuals as early as possible to preserve these cells.

Satya Dandekar's group has shown that initiating antiretroviral treatment very early in both SIV-infected macaques and in HIVinfected individuals results in much better (yet still incomplete) restoration of the intestinal $\mathrm{CD}^{+}{ }^{+} \mathrm{T}$ cell pool, as compared to individuals or macaques in whom therapy is delayed ${ }^{11,12}$. Others have shown that intestinal $\mathrm{CD} 4^{+} \mathrm{T}$ cells are never restored in individuals even after five years of therapy, and persistently undetectable viral loads in plasma ${ }^{5}$.

Finally, it is increasingly evident that vaccine strategies must target mucosal immune responses, and they must do so rapidly, as the virus eliminates the effector $\mathrm{CD} 4^{+} \mathrm{T}$ cells responsible for generating such within days of infection (Fig. 2). Unfortunately, the finding that memory $\mathrm{CD} 4^{+} \mathrm{T}$ cells are selectivity eliminated in primary infection is discouraging news for HIV vaccine development, as these cells are responsible for generating help for essentially all host immune responses. New approaches to vaccine concepts and design will likely therefore be needed to defeat this unique pathogen that rapidly and profoundly eliminates these crucial $\mathrm{CD}^{+}$ memory cells.

On a more positive note, memory cells express high levels of the CCR5 coreceptor, required for viral entry, and this feature may be exploited for prevention or treatment strategies. Many have recognized the significance of CCR 5 for HIV therapeutics, and several CCR5 inhibitors are currently in clinical trials. Targeting CCR5 expression in prevention strategies, such as vaccines and microbicides, is an emerging avenue of research that may prove to be our best hope for slowing the HIV epidemic.

1. Mattapallil, J.J. et al. Nature 434, 1093-1097 (2005).

2. Li, Q. et al. Nature 434, 1148-1152 (2005).

3. Veazey, R.S. et al. J. Virol. 74, 11001-11007 (2000).

4. Veazey, R.S. et al. J. Virol. 74, 57-64 (2000).

5. Mehandru, S. et al. Primary J. Exp. Med. 200 761-770 (2004)

6. Brenchley, J.M. et al. J. Exp. Med. 200, 749-759 (2004).

7. Veazey, R.S. et al. Science 280, 427-431 (1998).

8. Anton, P.A. et al. AIDS 17, 53-63 (2003).

9. Picker, L.J. et al. J. Exp. Med. 200, 1299-1314 (2004).

10. Lackner, A.A., Vogel, P., Ramos, R.A., Kluge, J.D. \& Marthas, M. Am. J. Pathol. 145, 428-439 (1994).

11. George, M.D., Reay, E., Sankaran, S. \& Dandekar, S. J. Virol. 79, 2709-2719 (2005).

12. Guadalupe, M. et al. J. Virol. 77, 11708-11717 (2003). 